\title{
Influence of Different Types of Recombination Active Defects on the Integral Electrical Properties of Multicrystalline Silicon Solar Cells
}

\author{
Dominik Lausch and Christian Hagendorf \\ Fraunhofer-Center für Silizium Photovoltaik CSP, Otto-Eißfeld-Straße 12, 06120 Halle (Saale), Germany \\ Correspondence should be addressed to Dominik Lausch; dominik.lausch@csp.fraunhofer.de
}

Received 17 October 2014; Revised 29 January 2015; Accepted 9 February 2015

Academic Editor: Haricharan S. Reehal

Copyright (C) 2015 D. Lausch and C. Hagendorf. This is an open access article distributed under the Creative Commons Attribution License, which permits unrestricted use, distribution, and reproduction in any medium, provided the original work is properly cited.

In this contribution the influence of different types of recombination-active defects on the integral electrical properties of multicrystalline $\mathrm{Si}$ solar cells is investigated. Based on a previous classification scheme related to the luminescence behavior of crystal defects, Type-A and Type-B defects are locally distinguished. It is shown that Type-A defects, correlated to iron contaminations, are dominating the efficiency by more than $20 \%$ relative through their impact on the short circuit current $I_{\mathrm{SC}}$ and open circuit voltage $V_{\mathrm{OC}}$ in standard Si material (only limited by recombination active crystal defects). Contrarily, Type-B defects show low influence on the efficiency of $3 \%$ relative. The impact of the detrimental Type-A defects on the electrical parameters is studied as a function of the block height. A clear correlation between the area fraction of Type-A defects and both the global Isc and the prebreakdown behavior (reverse current) in voltage regime-2 $(-11 \mathrm{~V})$ is observed. An outlier having an increased fullarea recombination activity is traced back to dense inter- and intragrain nucleation of Fe precipitates. Based on these results it is concluded that Type-A defects are the most detrimental defects in Si solar cells (having efficiencies > 15\%) and have to be prevented by optimized Si material quality and solar cell process conditions.

\section{Introduction}

Currently, the main challenges of the photovoltaic industry are to reduce the costs per watt peak and to increase the durability of the solar modules. To reduce the costs a considerable way is to use multicrystalline silicon (mc-Si) wafers. However, the efficiency of solar cells made of mc-Si wafers is lower compared to solar cells made of monocrystalline silicon wafers. A major reason for this is the presence of a large number of different recombination active defect structures in mc-Si materials. These defect structures have an influence on (i) the solar cell efficiency [1-3] and (ii) the electrical breakdown behavior and hence the reliability of the solar module [4]. In various publications the electrical properties of the solar cells and the presence of crystal defects were connected [5-8]. However, a promising connection was not performed so far since mc-Si solar cells suffer from a large number of crystal defects and not all crystal defects behave in the same way with respect to recombination and prebreakdown behavior. For a thorough analysis of the defects, it is necessary due to their large number and their different properties to classify the defect structures before investigating the causes and mechanisms of each class separately. Afterwards, the knowledge obtained can be applied for each defect class on solar cell level even on industrial scale.

In this contribution a further procedure for connecting crystal defects with the electrical properties is presented based on classified defect types. The local influence of different types of recombination active defects on the integral electrical properties of multicrystalline Si solar cells and blocks based on a systematic classification previously introduced [9] is investigated and discussed. For this, first, a short review of 
the classification will be given. Afterwards, the distribution of defects of various classes and the integrated electrical properties of the complete solar cells will be connected. Here the two main quality aspects of solar cells hence, the conversion efficiency and electrical prebreakdown behavior, will be in focus. It will be shown that in comparably low contaminated samples $(\eta>15 \%)$ a clear correlation between the area fraction of specific defect types and the electrical properties exists as long as no other limiting defects than recombination active crystal defects are present. Finally, the results will be discussed with respect to the current findings of the cause and the physical mechanisms of the corresponding defect types.

\section{Short Review of Defect Classification}

Despite the fact that defects in mc-Si solar cells have been investigated since several years [10-16], a systematic classification applicable on a macro- and microscopic scale is still missing. In previous publications $[9,17]$ we have introduced such a classification of recombination active defect structures in mc-Si solar cells using the band-to-band and subband luminescence as well as the prebreakdown behavior at crystal defects based on results published by Bothe et al. [18] and Schubert et al. [19]. One important characteristic of this classification is its simple and industry-related application due to the usage of standard electroluminescence methods opening the possibility to improve and control the solar cell process or using it for return issues.

In Figure 1 such a classification at a $6^{\prime \prime} \mathrm{mc}-\mathrm{Si}$ solar cell is shown as an example. The large number of recombination active defects can be located by their decreased EL signal shown in Figure 1(a). As a result of the defect classification procedure the lateral distribution of the distinguished defect classes is shown in Figure 1(b) color-coded. In summary, already on a macroscopic level ( 6 " solar cell) we can clearly distinguish two types of recombination active defects denoted as Type-A (purple) and Type-B (orange). Type-A defects are determined by low intensity of the indirect band-to-band luminescence at $1.1 \mathrm{eV}$, missing defect luminescence between 0.75 and $0.82 \mathrm{eV}$ and their so called type- 2 prebreakdown behavior [20]. Type-A defects are most likely caused by metal contaminations in form of precipitates and atomic decorations as reported in Lausch et al. [21]. Type-B defects are characterized by a decreased band-to-band luminescence, high intensity of defect luminescence, and absence of type2 prebreakdown behavior. The responsible contamination could not be determined so far, but results are pointing towards a possible influence of the process of the $\mathrm{SiN}$ layer formation [22]. On a microscopic scale and at room temperature all recombination active defects were found at line defects which are most likely grain boundaries. The general type of recombination active defects is unclear so far and is under discussion [23]. Therefore no connection to small angle grain boundaries or other grain boundary types can be drawn so far. In particular, Type-A defects seem to be characterized by a broad range of misorientation angles as shown recently [24]. But single dislocations within the inner grain region do not seem to be detrimental to the electrical properties [25].

\section{Experimentals and Methods}

The samples analyzed are silicon solar cells fabricated with a standard commercial process at the company Q-Cells SE in 2009 based on B-doped (acceptor concentration $N_{A} \sim$ $10^{16} \mathrm{~cm}^{-3}$ ) $156 \times 156 \mathrm{~mm}^{2}$ multicrystalline silicon (mc-Si) wafers made from standard electronic grade silicon with high purity. The material was crystalized in a standard G5 crucible. For wafering an edge block was chosen. Every 10th solar cell was screen-printed with another layout in order to cut out mini solar cells with size of $1 \times 1 \mathrm{~cm}^{2}$ for detailed investigations. The cutting was performed by an optimized laser process with very low influence on the shunt resistance. Spectrally integrated electroluminescence images under forward [26] EL and reverse bias [27] ReBEL were acquired using a common Si CCD array. The defect luminescence imaging procedure (ELsub) is described in [28].

In Figure 2 a schematic representation of the procedure applied in this work to correlate the lateral distribution of defect types with the globally integrated electrical properties of the solar cells is shown. The area fraction of the classified defects is extracted from the color-coded defect distribution map which is the result of the classification procedure by simply counting out the pixels of the specific defect type divided by all pixels of the full area. The area fraction correlates to the amount of the corresponding defect type and is given in \%. The global electrical properties, that is, $V_{\mathrm{OC}}, I_{\mathrm{SC}}$, $\eta$, and reverse current $I_{r}$, of the solar cells are measured in line during solar cell production at the company Q-Cells SE. The electrical properties of the mini solar cells were acquired by a lab sun simulator. Finally, the area fraction of the defect classes is correlated with the electrical properties.

\section{Influence of the Defect Types on the Electrical Characteristic}

\subsection{Experimental Results}

4.1.1. Estimation of the Influence of the Defect Types on the Local Electrical Parameters by Using Mini Solar Cells. To estimate the influences of Type-A and Type-B defects, respectively, on the electrical properties of the solar cell, three mini solar cells $\left(1 \times 1 \mathrm{~cm}^{2}\right)$ out of a $6^{\prime \prime}$ solar cell were prepared having a large number of Type-A defects and Type-B defects and a sample having a low number of recombination active defects according to an EL measurement. The separation procedure is not influencing significantly $V_{\mathrm{OC}}, I_{\mathrm{SC}}$, or $\eta$ (except a slight decrease of the parallel resistance). This investigation at one solar cell shall just give first evidence to the influence of the different defect types for a further focusing. The positions of the mini solar cells are marked in the EL image in Figure 3 with a purple rectangle (sample with Type-A defects), an orange rectangle (sample with TypeB defects), and a black rectangle (reference sample with low 


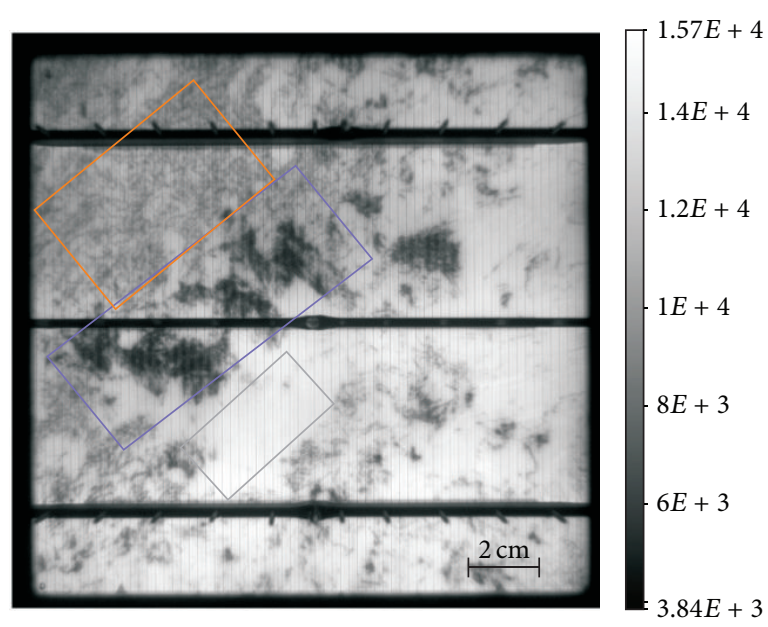

Type-A

Type-B

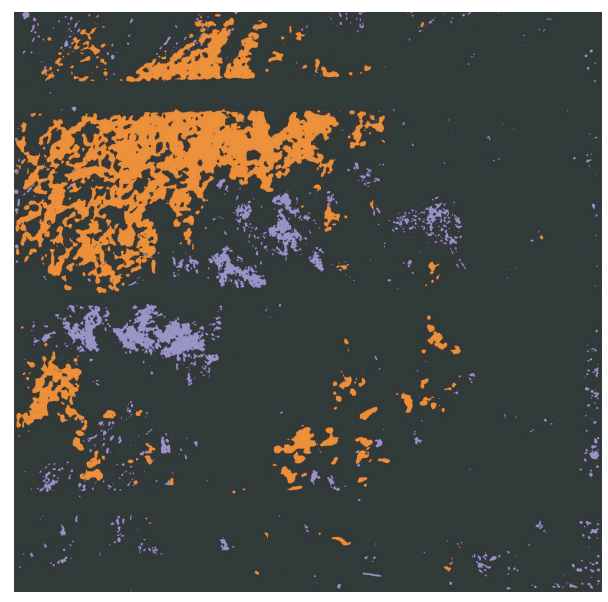

Type-A

Type- $B$

(a)

(b)

FIGURE 1: (a) Electroluminescence image (EL) of a mc-Si solar cell. The recombination active defects (visible due to their decreased EL signal) can be separated by an image processing procedure described in [9] into the lateral distribution of Type-A (purple) and Type-B (orange) defects shown in (b).
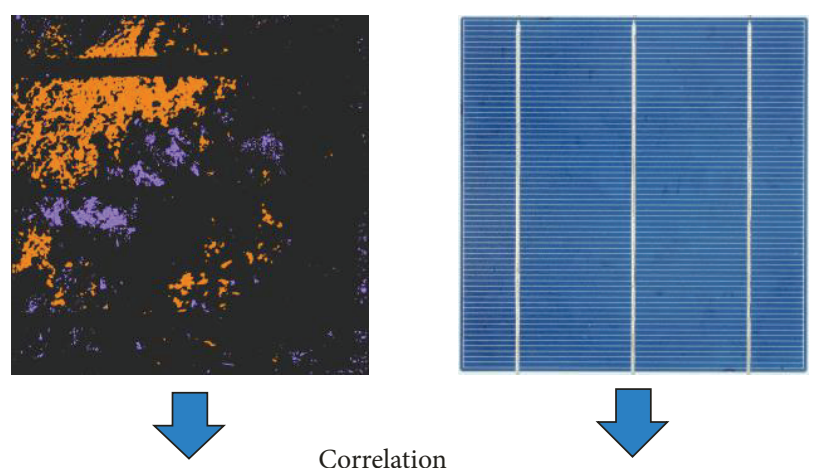

Correlation

Area fraction of

Type-A, Type-B

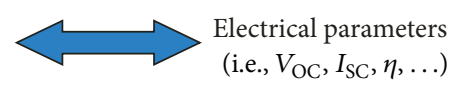

Type-A

Type-B

Figure 2: Schematic illustration of the procedure to correlate the area fraction of different recombination active defects with the globally integrated electrical parameter of the solar cell. The area fraction of the defect types is extracted from the color-coded distribution of the defect types. The electrical properties are measured in line during solar cell production.

defect density) according to the previously introduced color code. The solar cell parameters of each mini solar cell were measured by means of a sun simulator under 1 sun in the lab. The results are summarized in the table in Figure 3. The efficiency results are normalized to the reference sample for a relative comparison.

Type-A defects have the strongest detrimental impact on the efficiency $\eta$ of the mini solar cells. The efficiency is lowered by $20 \%$ relative caused by a decreased $V_{\mathrm{OC}}$ and $I_{\mathrm{SC}}$ compared to the reference sample. The sample is not fully covered by Type-A defects; hence the result is even underestimating the influence of Type-A defects. In contrast to this, Type-B defects show a clearly lower influence of about $3 \%$ efficiency decrease compared to the reference sample. Both Type-A and Type-B defects are decreasing $I_{\mathrm{SC}}$ as well as $V_{\mathrm{OC}}$ of the mini solar cell. The fill factors of the mini solar cells are comparable and not substantially influenced by the defect types. A more adequate valuation of the influences of the defect types on the electrical parameters is a discussion about the two-diode model, hence J01 and J02 currents. However, this was not done so far and will be performed in an upcoming contribution. Nevertheless first results clearly show that Type-A defects primarily influence J01.

It can be concluded that Type-B defects have only a relatively low influence on the overall solar cell efficiency and Type-A defects are detrimental. Therefore, Type-A defects will be in focus in the following.

4.1.2. Block-Height Dependent Analysis of Type-A Defects in Correlation to the Integral Solar Cell Properties. In Figures 4(a), 4(b), and 4(c) the area fraction of Type-A defects and the electrical properties of every 30 th $6^{\prime \prime}$ solar cell taken out of the Si block at different block positions are correlated. Figure 4(d) shows a comparison of the area fraction of Type-A defects and $I_{\mathrm{SC}}$ as a function of the wafer number from the bottom to the top region of a Si block (higher wafer number represents higher block position).

In agreement with results of Section 4.1, the efficiency is decreasing with increasing area fraction of Type-A defects as can be seen in Figure 4(a). A decreasing efficiency with increasing area fraction of Type-B defects cannot be obtained 


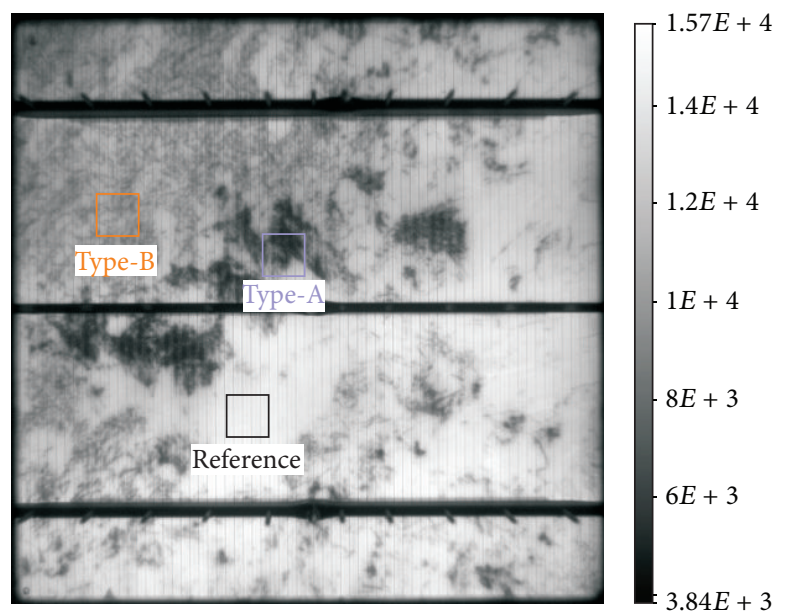

\begin{tabular}{|c|c|c|c|}
\hline & Reference & Type-A & Type-B \\
\hline $\begin{array}{c}\text { Normalized } \\
\text { efficiency } \eta\end{array}$ & 1 & 0.8 & 0.97 \\
\hline $\begin{array}{c}\text { Short circuit } \\
\text { current } \\
J_{\text {SC }}\left(\mathrm{mA} / \mathrm{cm}^{2}\right)\end{array}$ & 34 & 32 & 33.5 \\
\hline $\begin{array}{c}\text { Open circuit } \\
\text { voltage } \\
V_{\mathrm{OC}}(\mathrm{mV})\end{array}$ & 618.6 & 566.7 & 593.7 \\
\hline $\begin{array}{c}\text { Power } \\
P\left(\mathrm{~mW} / \mathrm{cm}^{2}\right)\end{array}$ & 18.4 & 15.2 & 17.6 \\
\hline
\end{tabular}

FIGURE 3: EL image of a $6^{\prime \prime} \mathrm{mc}$-Si solar cell. The mini solar cells which were taken are marked with rectangles. The sample with a large number of Type-A defects is marked with a purple rectangle, the sample with Type-B defects with an orange rectangle, and the sample with a low defect density (reference) with a black rectangle. In the table the main results of the sun simulator measurements of the mini solar cells are summarized. The efficiencies are normalized to the sample with the low defect density (reference sample).

(shown in [8]). The efficiency loss in Figure 4(a) seems to saturate to higher area fractions of Type-A defects $(>8 \%)$ for the investigated samples. $V_{\mathrm{OC}}$ is decreasing with increasing area fraction of Type-A defects as well (see Figure 4(b)) but at a specific point $V_{\mathrm{OC}}$ slightly increases to higher area fractions of Type-A defects. A rather linear correlation with the area fraction of Type-A defects is observed for $I_{\mathrm{SC}}$ (Figure 4(c)). With increasing area fraction of Type-A defects $I_{\mathrm{SC}}$ is decreasing linearly guided by the red dotted line in Figure 4(c) which is a linear approximation. An exception to this trend is the sample REF-37 (marked with a red dotted circle in Figure 4) which was cut out from the very bottom region close to the crucible wall. REF-37 shows low $I_{\mathrm{SC}}$ and $\eta$ values at low area fraction of Type-A defects.

In order to show that the correlation of $I_{\mathrm{SC}}$ and the area fraction of Type-A defects holds for the whole Si block, in Figure 4(d) both $I_{\mathrm{SC}}$ and the area fraction of Type-A defects are plotted as a function of the block height from bottom (low wafers numbers) to top (high wafer numbers). Note the area fraction of Type-A defects is plotted from high to low values (\%) in order to visualize the correlation. The area fraction of Type-A defects is increasing towards the top of the Si block. Contrarily, $I_{\mathrm{SC}}$ is decreasing towards the top of the Si block. Nevertheless, it is obvious that both show rather the same trend. Hence with an increase of Type-A defects $I_{\mathrm{SC}}$ is decreasing over the whole Si block. Therefore, despite the fact that you can localize Type-B defects with EL measurements quite clear, their influence on the electrical properties seems rather low. Deviations are caused by measurement inaccuracies or additional influences on $I_{\mathrm{SC}}$. Therefore, it can be concluded that the trend of the electrical properties within the investigated mc-Si block is dominated by the area fraction of Type-A defects over the whole block height. Note that this is only the case because this block is of high quality and not influenced by other limiting defects than recombination active defects.

\subsection{Discussion of the Electrical Properties}

4.2.1. Discussion about the Influence of the Defect Types on the Integral Electrical Properties. Type-A defects are mainly dominating the solar cell efficiency through their detrimental impact on the $I_{\mathrm{SC}}$ and $V_{\mathrm{OC}}$ caused by recombination of generated minority carriers at these defect structures. The reasons for the strong recombination activity of Type-A defects are metal silicide precipitates and most likely metal decorated structural defects in the vicinity of these precipitates as shown in [21]. These impurities are introducing various defect levels within the band gap increasing the recombination probability for minority carriers as discussed in [21]. Most likely the iron is diffused out of the crucible into the material [29].

Type- $B$ defects have no significant influence on the electrical properties of solar cells if a large number of TypeA defects is present. However, Type- $B$ defects are reducing strongly the indirect band-to-band luminescence at $1.1 \mathrm{eV}$ [30] leading often to the wrong assumption of a stronger negative influence on the efficiency by Type- $\mathrm{B}$ defects. The low influence of Type-B defects is confirmed by investigations of solar cells made of various silicon feedstock, that is feedstock with different contamination levels and upgraded metallurgical silicon material [25] and by Bakowskie et al. [8] who have investigated the influence of different defect types on both wafer and solar cell level. Additionally, they have shown an activation of regions with Type-A defects by the solar cell process most likely due to a dissolving of iron precipitates and a consecutive gettering during cool-down at the grain boundary planes. Detailed root cause analyses on microstructural level of Type-A and Type-B defects including a discussion about the physical origin of the recombination processes can be found elsewhere [21, 22, 31].

4.2.2. Discussion about the Block-Height Dependent Integral Electrical Properties. $I_{\mathrm{SC}}$ is decreasing linearly with increasing number of Type-A defects (Figure 4(c)) since the more 


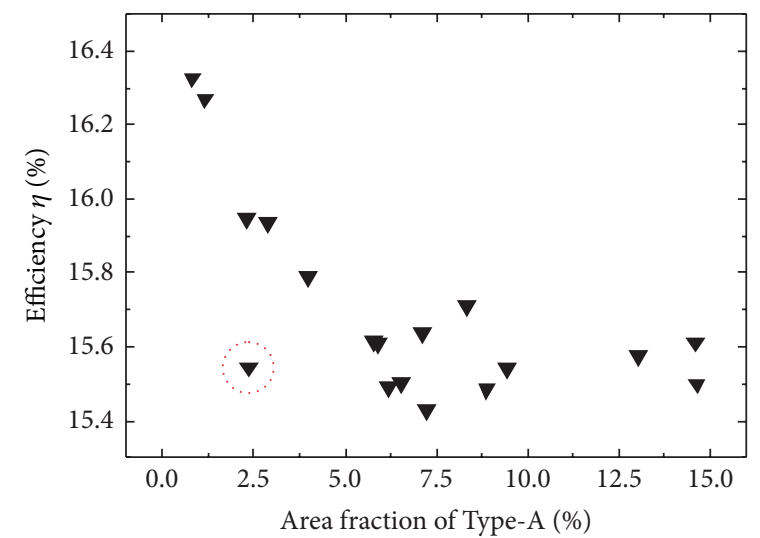

Efficiency $\eta$

(a)

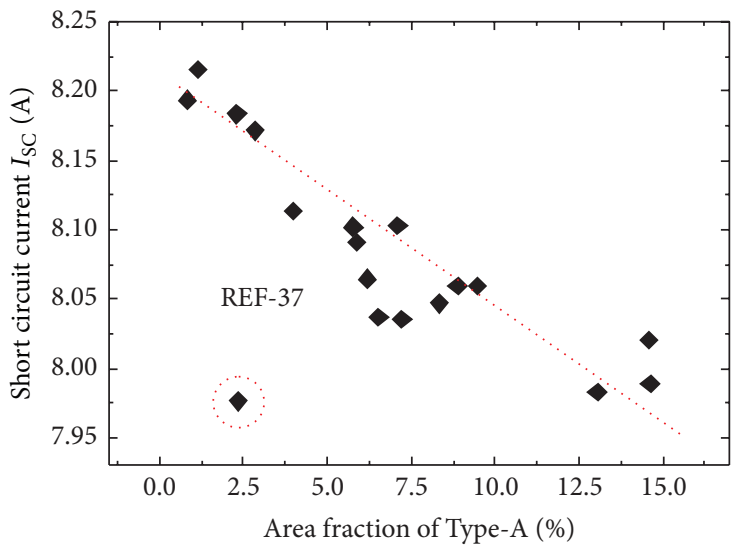

Short circuit current $I_{\mathrm{SC}}$

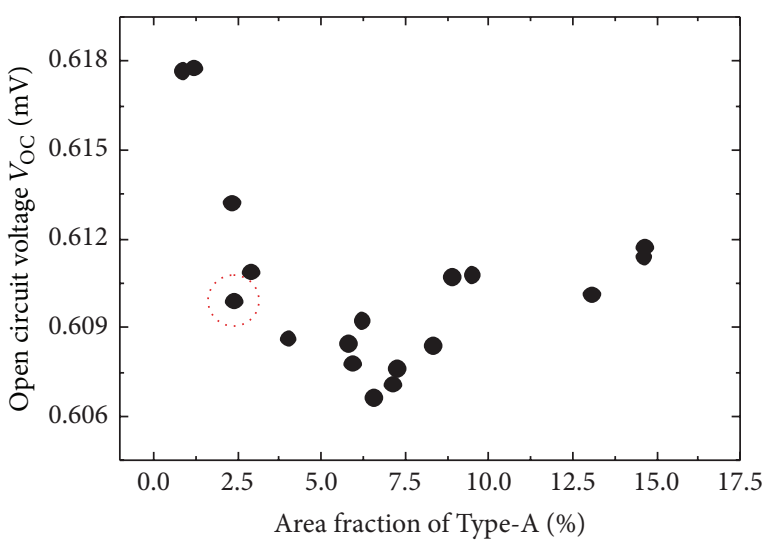

Open circuit voltage $V_{\mathrm{OC}}$

(b)

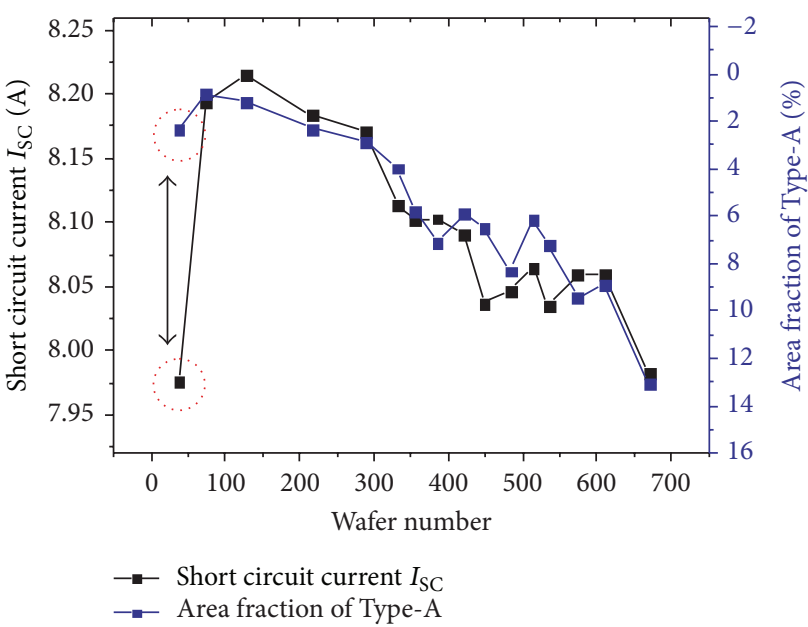

(d)

FIGURE 4: (a) Efficiency $\eta$, (b) open circuit voltage $V_{\mathrm{OC}}$, and (c) short circuit current $I_{\mathrm{SC}}$ as a function of the area fraction of Type-A defects of 6 " silicon solar cells. In (d) the $I_{\mathrm{SC}}$ and the area of fraction of Type-A defects are plotted in dependence on the wafer number identifying the ingot position from bottom to top of the silicon block. In all figures the sample REF-37 is marked with a red circle.

the recombination active defects per area, the higher the recombination probability for generated minority carriers. The recombination probability at Type-A defects is quite high since the recombination takes place via a high concentration of midgap defect levels as discussed. Deviations can be caused by further influences on $I_{\mathrm{SC}}$, that is, reflection losses. $V_{\mathrm{OC}}$ and consequently $\eta$ saturate with higher number of Type-A defects. The solar cells with slightly increasing $V_{\mathrm{OC}}$ are cut out from the upper part of the Si block. We assume that the increasing $V_{\mathrm{OC}}$ is caused by the segregation of boron to the top leading to an increasing $V_{\text {OC }}$ with increasing block height. Subsequently, this leads to a weakened decrease of $\eta$ too.

Obviously, Type-A defects are dominating the trend of the integral electrical properties significantly over the whole $\mathrm{Si}$ block. However, the general baseline of the electrical properties is of course given by the solar cell process quality. Note that this holds only for high quality material which is only dominated by recombination active defects at crystal defects; that is, a "full-area" recombination activity taking place also in the inner grain regions can cover these Type-A defects as it is the case for REF-32. Specifically, in highly contaminated samples with $\eta \ll 14 \%$, a full-area recombination activity is often present.

\section{Influence of the Defect Types on the Electrical Breakdown Behavior}

5.1. Influence of Type-A Defects on the Prebreakdown Behavior in Dependence on the Block Height. A further important property of solar modules is their reliability. This can be negatively influenced by prebreakdown processes and consecutive heating [32] which can lead even to destruction of solar modules since the rear side foil of a module is quite sensitive to heat. In Figure 5(a) the reverse current $I_{r}$ at a reverse voltage of $V_{r}=11 \mathrm{~V}$ (left axis) and the area fraction of Type-A defects (right axis) as a function of the wafer number are shown. At a base doping concentration of about $N_{A} \sim$ $10^{16} \mathrm{~cm}^{-3}$ the reverse voltage of $11 \mathrm{~V}$ is in the middle range 


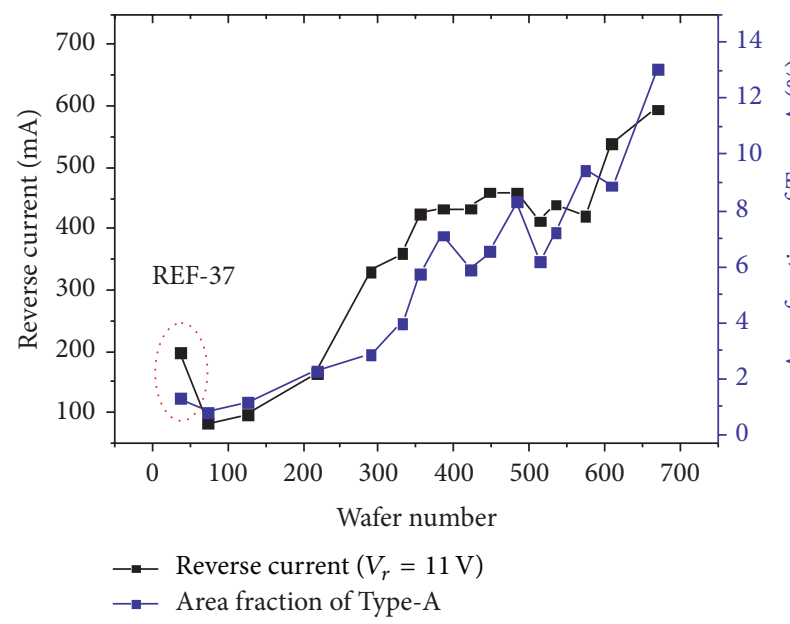

(a)

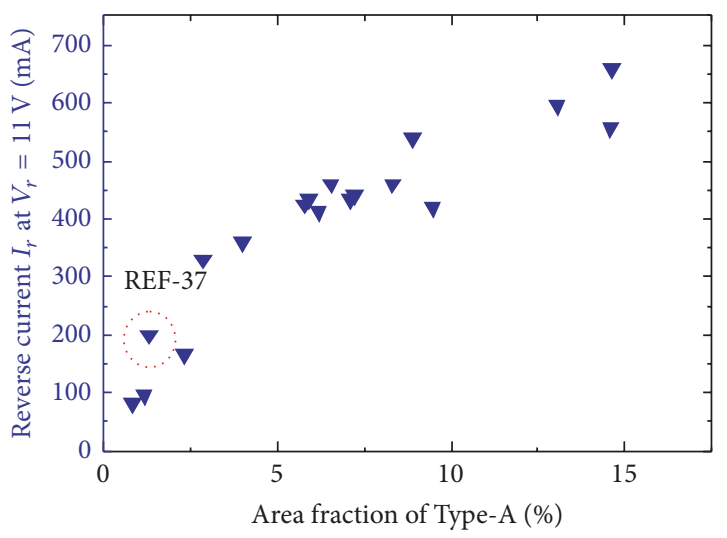

(b)

FiguRE 5: (a) Reverse current at $V_{r}=11 \mathrm{~V}$ and area fraction of Type-A defects as a function of the wafer number. A correlation between the number of Type-A defects and the reverse current at $-11 \mathrm{~V}$ can be seen. In (b) the area fraction of Type-A defects in dependence on the reverse current at $V_{r}=11 \mathrm{~V}$ is shown.

of the reverse voltage regime- $2[9,33]$ which is dominated by the so-called type-2 prebreakdown sites [20] and defined by an exponential increased reverse $I-V$ characteristic from about $-7 \mathrm{~V}$ to $-12 \mathrm{~V}$. With increasing number of Type-A defects the reverse current is increasing too. Therefore, TypeA defects are dominating the prebreakdown behavior in reverse voltage regime-2. Small deviations can be caused by additional contributions to the reverse current like different edge isolations of the solar cells, type-1 prebreakdown sites [34], ohmic shunts [35], or simply the inaccuracy of the Type-A defect determination. In Figure 5(b) $I_{r}$ at $V_{r}=11 \mathrm{~V}$ in dependence on the area fraction of Type-A defects is shown. $I_{r}$ is increasing with increasing area fraction of TypeA defects. Type-B defects do not show any prebreakdown behavior in voltage regime-2; thus Type- $\mathrm{B}$ defects are not influencing the reverse characteristic in voltage regime- 2 of the solar cell (not shown here).

\subsection{Discussion about the Prebreakdown Behavior at Type-A} Defects Caused by Iron Precipitates. In Figure 5 an increasing reverse current $I_{r}$ at voltage regime-2 $(-11 \mathrm{~V})$ with increasing area fraction of Type-A defects can be observed which is expected since one characteristic of Type-A defects is their type- 2 prebreakdown behavior. This type- 2 prebreakdown is caused by iron precipitates located at Type-A defects [21]. These iron precipitates are quasi-metallic $\alpha-\mathrm{FeSi}_{2}$ precipitates forming a Schottky junction where thermionic field emission under reverse bias occurs as described in [31]. Exactly at these positions ReBEL is emitted [27]. Hence, iron precipitates can be located by their ReBEL signal. The number of TypeA defects and hence type-2 prebreakdown sites and the prebreakdown current is increasing with block height as shown in Figure 5(a). As shown Type-B defects do not show a prebreakdown behaviour in voltage regime-2. However, by further increase of the reverse voltage up to voltage regime- 3
( $>14 \mathrm{~V}$ ), Type-B defects show type-3 prebreakdown behavior like all structural defects due to curved $\mathrm{p}-\mathrm{n}$ junctions caused by etch pits [36] or preferred phosphorus diffusion along defect lines [37] leading to a field enhancement at the $\mathrm{p}-\mathrm{n}$ junction up to a level where an avalanche breakdown occurs [38].

\section{The Outlier Solar Cell REF-37}

An exception to the results shown in this contribution is the solar cell REF-37. REF-37 was cut out of the very bottom region of the Si block at about $8 \mathrm{~mm}$. The silicon block used in this investigation was cropped at only $5 \mathrm{~mm}$ at bottom region to investigate the influence of high contaminations rather than the common $15 \mathrm{~mm}$. An EL measurement of this solar cell can be seen in Figure 6(a). It is obvious that the EL signal is not dominated by recombination active defects but by a full-area recombination influence. Here, most likely a large amount of iron is present diffused into the crystal out of the crucible up to a concentration of $10^{16} \mathrm{~cm}^{-3}$ [29]. This is proven by the ReBEL image at voltage regime-2 in Figure 6(b). Here a dense distribution of type2 prebreakdown sites can be observed. As discussed type- 2 prebreakdown sites are caused by Fe precipitates.

So it can be concluded that REF-37 suffers from a large amount of iron contamination which is precipitated or atomic in the inner grain region. This leads to a strongly decreased lifetime over the whole area in particular also in the inner grain regions [39]. The iron precipitates are decreasing the lifetime due to minority carrier recombination at the interface of silicon and iron significantly leading to the low EL signal over the whole area (also in the inner grain regions) in the EL image in Figure 6(a) explaining the missing correlation of Type-A defects and the solar cell parameters as marked in Figures 3 and 4 . However, near a grain boundary the lifetime 


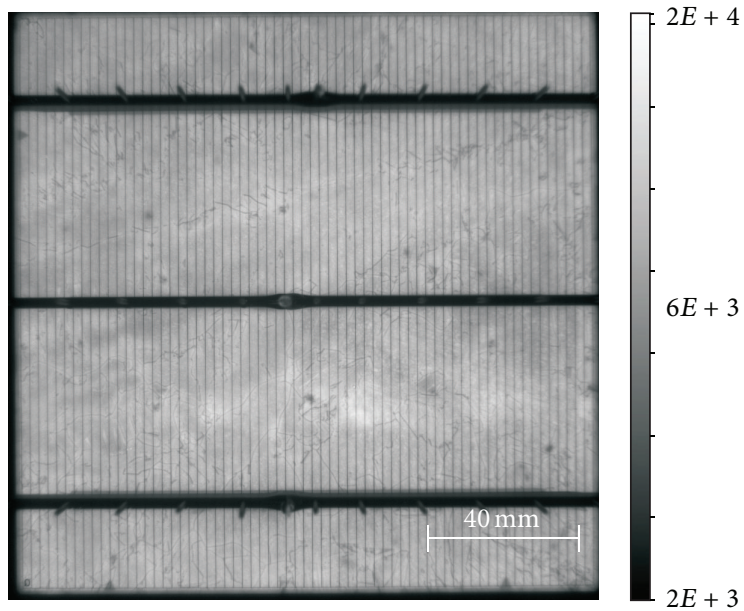

(a)

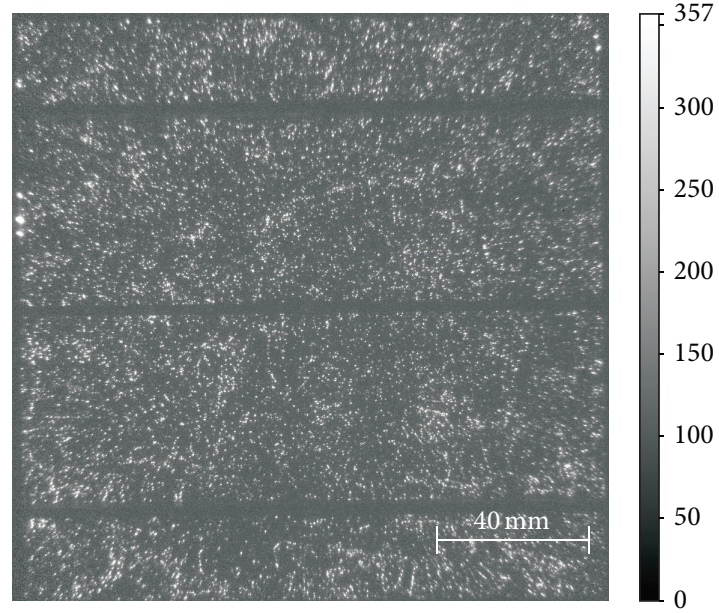

(b)

Figure 6: (a) EL image at forward bias $(V \sim 0.6 \mathrm{~V})$ of REF-37. The EL signal is decreased over the whole area. (b) ReBEL image at voltage regime-2 $\left(V_{r}=11 \mathrm{~V}\right)$. Single prebreakdown sites distributed over the whole solar cell area (independent of grain boundaries) can be observed due to their spot-like occurrence.

is increased due to gettering to the grain boundary planes leading to a slightly increased EL signal in the vicinity of grain boundaries [40].

\section{Summary}

In this contribution the local influence of different types of recombination-active defects on the integral electrical properties of multicrystalline $\mathrm{Si}$ solar cells and blocks was investigated. Based on a previous classification scheme related to band-to-band and subband luminescence as well as prebreakdown behavior Type-A and Type-B defects were locally distinguished on 6 " solar cell level.

It was shown that Type-A defects, which are correlated to iron contaminations, are dominating the efficiency through their impact on the local $I_{\mathrm{SC}}$ and $V_{\mathrm{OC}}$ of the solar cell. TypeA defects lower the efficiency of mini solar cells by more than $20 \%$ relative whereas Type-B defects show only a low influence on the local efficiency of $\sim 3 \%$ relative despite the fact that Type-B defects can be localized well in EL imaging often leading to the wrong assumption that Type-B defects are detrimental as well.. The impact of Type-A defects on the integral electrical solar cell parameters is studied as a function of the block height. A clear correlation between the area fraction of Type-A defects and the integral solar cell parameters is observed. Furthermore, the influence of the area fraction of Type-A defects on the integral solar cell prebreakdown behavior is analyzed as a function of the block height. The prebreakdown behavior (reverse current) in voltage regime-2 $(\sim-11 \mathrm{~V})$ is mainly driven by Type-A defects. In contrast, Type-B defects do not show any influence on the prebreakdown behavior in voltage regime-2 $(\sim-11 \mathrm{~V})$ at all. Additionally, beside the strong local impact of Type-A defects an increased full-area recombination activity in REF37 is found at the very bottom of the Si block which is proved to be related to dense inter- and intragrain nucleation of
Fe precipitates diffused out of the crucible wall by ReBEL imaging.

Based on these results it is concluded that Type-A defects are the most detrimental defects in high quality $\mathrm{Si}$ solar cells $(\eta>15 \%)$ and have to be prevented by optimized $\mathrm{Si}$ material quality and solar cell process conditions. Therefore, the classification of recombination active defects is useful to gain causes for decreased solar cell efficiency consecutively to improve the material quality or the solar cell process on an industrial scale.

\section{Conflict of Interests}

The authors declare that there is no conflict of interests regarding the publication of this paper.

\section{Acknowledgment}

The samples investigated were provided by the company QCells SE in 2009. A lot of support by Q-Cells employees is gratefully acknowledged. In particular, Kai Petter, Ronny Bakowskie, and Ramona Weiske are gratefully acknowledged for various support.

\section{References}

[1] J. D. Zook, "Effects of grain boundaries in polycrystalline solar cells," Applied Physics Letters, vol. 37, no. 2, pp. 223-226, 1980.

[2] A. A. Istratov and E. R. Weber, "Electrical properties and recombination activity of copper, nickel and cobalt in silicon," Applied Physics A: Materials Science \& Processing, vol. 66, no. 2, pp. 123-136, 1998.

[3] M. Kittler, W. Seifert, and O. Krüger, "Electrical behaviour of crystal defects in silicon solar cells," Diffusion and Defect Data B: Solid State Phenomena, vol. 78-79, pp. 39-48, 2001. 
[4] O. Breitenstein, J. Bauer, K. Bothe et al., "Understanding junction breakdown in multicrystalline solar cells," Journal of Applied Physics, vol. 109, no. 7, Article ID 071101, 2011.

[5] S. Pizzini, A. Sandrinelli, M. Beghi et al., "Influence of extended defects and native impurities on the electrical properties of directionally solidified polycrystalline silicon," Journal of the Electrochemical Society, vol. 135, no. 1, pp. 155-156, 1988.

[6] M. Turek and D. Lausch, "Solar cell performance prediction using advanced analysis methods on optical images of as-cut wafers," Energy Procedia, vol. 38, pp. 190-198, 2013.

[7] S. Rißland and O. Breitenstein, "High resolution saturation current density imaging at grain boundaries by lock-in thermography," Solar Energy Materials and Solar Cells, vol. 104, pp. 121-124, 2012.

[8] R. Bakowskie, R. Lantzsch, T. Kaden et al., "Comparison of recombination active defects in multicrystalline silicon by means of photoluminescence imaging and reverse biased electroluminescence," in Proceedings of the 26th European Photovoltaic Solar Energy Conference and Exhibition, pp. 1839-1842, 2010.

[9] D. Lausch, R. Bakowskie, M. Lorenz, S. Schweizer, K. Petter, and C. Hagendorf, "Classification of recombination-active defects in multicrystalline solar cells made from upgraded metallurgical grade (UMG) silicon," in Solid State Phenomena, vol. 178-179, pp. 88-93, Trans Tech Publications, 2011, http://www.scientific.net/SSP.178-179.88.

[10] S. Steingrube, O. Breitenstein, K. Ramspeck, S. Glunz, A. Schenk, and P. P. Altermatt, "Explanation of commonly observed shunt currents in c-Si solar cells by means of recombination statistics beyond the Shockley-Read-Hall approximation," Journal of Applied Physics, vol. 110, no. 1, Article ID 014515, 2011.

[11] H. C. Card and E. S. Yang, "Electronic processes at grain boundaries in polycrystalline semiconductors under optical illumination," IEEE Transactions on Electron Devices, vol. 24, no. 4, pp. 397-402, 1977.

[12] W. Seifert, G. Morgenstern, and M. Kittler, "Influence of dislocation density on recombination at grain boundaries in multicrystalline silicon," Semiconductor Science and Technology, vol. 8, no. 9, pp. 1687-1691, 1993.

[13] V. Kveder, M. Kittler, and W. Schröter, "Recombination activity of contaminated dislocations in silicon: a model describing electron-beam-induced current contrast behavior," Physical Review B-Condensed Matter and Materials Physics, vol. 63, no. 11, Article ID 115208, 2001.

[14] J. P. Hirth, "The influence of grain boundaries on mechanical properties," Metallurgical Transactions, vol. 3, no. 12, pp. 30473067, 1972.

[15] C. H. Seager, "Grain boundary recombination: theory and experiment in silicon," Journal of Applied Physics, vol. 52, no. 6, pp. 3960-3968, 1981.

[16] R. Bakowskie, G. Kesser, R. Richter et al., "Fast method to determine the structural defect density of $156 \times 156 \mathrm{~mm}^{2} \mathrm{Mc}-$ Si wafers," Energy Procedia, vol. 27, pp. 179-184, 2012.

[17] D. Lausch, K. Petter, B. Henke, R. Bakowskie, S. Schweizer, and C. Hagendorf, "Classification of recombination active defect structures in multicrystalline silicon solar cells," Energy Procedia, vol. 8, pp. 28-34, 2011.

[18] K. Bothe, K. Ramspeck, D. Hinken et al., "Luminescence emission from forward- and reverse-biased multicrystalline silicon solar cells," Journal of Applied Physics, vol. 106, no. 10, Article ID 104510, 2009.
[19] M. C. Schubert, W. Kwapil, J. Schön et al., "Analysis of performance limiting material properties of multicrystalline silicon," Solar Energy Materials and Solar Cells, vol. 94, no. 9, pp. 14511456, 2010.

[20] W. Kwapil, P. Gundel, M. C. Schubert et al., "Observation of metal precipitates at prebreakdown sites in multicrystalline silicon solar cells," Applied Physics Letters, vol. 95, no. 23, Article ID 232113, 2009.

[21] D. Lausch, K. Petter, R. Bakowskie, J. Bauer, O. Breitenstein, and C. Hagendorf, "Classification and investigation of recombination-active defect structures in multicrystalline silicon solar cells-recombination models," in Proceedings of the 27th European Photovoltaic Solar Energy Conference and Exhibition (EUPVSEC '12), pp. 723-728, Frankfurt, Germany, 2012, https://www.eupvsec-proceedings.com/proceedings/ checkout.html? paper $=17019$.

[22] S. Johnston, H. Guthrey, F. Yan et al., "Correlating multicrystalline silicon defect types using photoluminescence, defectband emission, and lock-in thermography imaging techniques," IEEE Journal of Photovoltaics, vol. 4, no. 1, pp. 348-354, 2014.

[23] D. Oriwol, E.-R. Carl, A. N. Danilewsky et al., "Small-angle subgrain boundaries emanating from dislocation pile-ups in multicrystalline silicon studied with synchrotron white-beam X-ray topography," Acta Materialia, vol. 61, no. 18, pp. 69036910, 2013.

[24] D. Lausch, M. Gläser, and C. Hagendorf, "Determination of crystal grain orientations by optical microscopy at textured surfaces," Journal of Applied Physics, vol. 114, no. 19, Article ID 194509, 2013.

[25] D. Lausch, Einfluss struktureller Defekte auf die elektrischen Eigenschaften multikristalliner Silizium-Solarzellen [Dissertationsarbeit], Martin-Luther Universität Halle-Wittenberg, Mathematisch-Naturwissenschaftlich-Technischen Fakultät, Halle, Germany, 2013.

[26] T. Fuyuki, H. Kondo, T. Yamazaki, Y. Takahashi, and Y. Uraoka, "Photographic surveying of minority carrier diffusion length in polycrystalline silicon solar cells by electroluminescence," Applied Physics Letters, vol. 86, no. 26, Article ID 262108, 2005.

[27] D. Lausch, K. Petter, H. von Wenckstern, and M. Grundmann, "Correlation of pre-breakdown sites and bulk defects in multicrystalline silicon solar cells," Physica Status Solidi-Rapid Research Letters, vol. 3, no. 2-3, pp. 70-72, 2009.

[28] D. Lausch and C. Hagendorf, "Microscopic study of defect luminescence between $0.72-0.85 \mathrm{eV}$ by optical microscopy," Microscopy Research, vol. 2, no. 1, pp. 9-12, 2014.

[29] M. C. Schubert, J. Schon, F. Schindler et al., "Impact of impurities from crucible and coating on mc-silicon quality-The example of iron and cobalt," IEEE Journal of Photovoltaics, vol. 3, no. 4, pp. 1250-1258, 2013.

[30] H. Guthrey, S. Johnston, F. Yan, B. Gorman, and M. Al-Jassim, "Defect band luminescence intensity reversal as related to application of anti-reflection coating on mc-Si PV Cells," in Proceedings of the 38th IEEE Photovoltaic Specialists Conference (PVSC '12), pp. 227-230, IEEE, Austin, Tex, USA, June 2012.

[31] A. Hähnel, J. Bauer, H. Blumtritt, O. Breitenstein, D. Lausch, and W. Kwapil, "Electron microscope verification of prebreakdowninducing $\alpha$-FeSi 2 needles in multicrystalline silicon solar cells," Journal of Applied Physics, vol. 113, no. 4, Article ID 044505, 2013.

[32] A. Woyte, J. Nijs, and R. Belmans, "Partial shadowing of photovoltaic arrays with different system configurations: literature 
review and field test results," Solar Energy, vol. 74, no. 3, pp. 217233, 2003.

[33] W. Kwapil, M. Wagner, M. C. Schubert, and W. Warta, "High net doping concentration responsible for critical diode breakdown behavior of upgraded metallurgical grade multicrystalline silicon solar cells," Journal of Applied Physics, vol. 108, no. 2, Article ID 023708, 2010.

[34] D. Lausch, K. Petter, R. Bakowskie et al., "Identification of pre-breakdown mechanism of silicon solar cells at low reverse voltages," Applied Physics Letters, vol. 97, no. 7, Article ID 073506, 2010.

[35] O. Breitenstein, J. P. Rakotoniaina, M. H. Al Rifai, and M. Werner, "Shunt types in crystalline silicon solar cells," Progress in Photovoltaics: Research and Applications, vol. 12, no. 7, pp. 529-538, 2004.

[36] J. Bauer, J.-M. Wagner, A. Lotnyk et al., "Hot spots in multicrystalline silicon solar cells: avalanche breakdown due to etch pits," Physica Status Solidi (RRL)_Rapid Research Letters, vol. 3, no. 2-3, pp. S.40-S.42, 2009.

[37] J. Bauer, D. Lausch, H. Blumtritt, N. Zakharov, and O. Breitenstein, "Avalanche breakdown in multicrystalline solar cells due to preferred phosphorous diffusion at extended defects," Progress in Photovoltaics: Research and Applications, vol. 21, no. 7, pp. 1444-1453, 2013.

[38] A. G. Chynoweth and K. G. McKay, "Photon emission from avalanche breakdown in silicon," Physical Review, vol. 102, no. 2, pp. 369-376, 1956.

[39] J. Schön, A. Haarahiltunen, H. Savin et al., "Analyses of the evolution of iron-silicide precipitates in multicrystalline silicon during solar cell processing," IEEE Journal of Photovoltaics, vol. 3, no. 1, pp. 131-137, 2013.

[40] J. Lu, M. Wagener, G. Rozgonyi, J. Rand, and R. Jonczyk, "Effects of grain boundary on impurity gettering and oxygen precipitation in polycrystalline sheet silicon," Journal of Applied Physics, vol. 94, no. 1, pp. 140-144, 2003. 


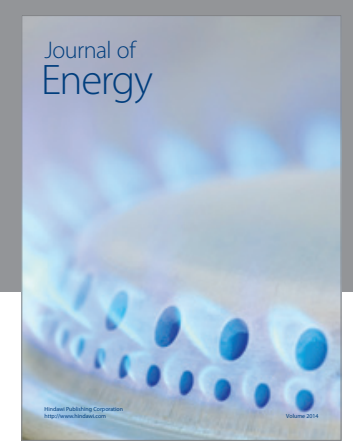

Journal of

Industrial Engineering
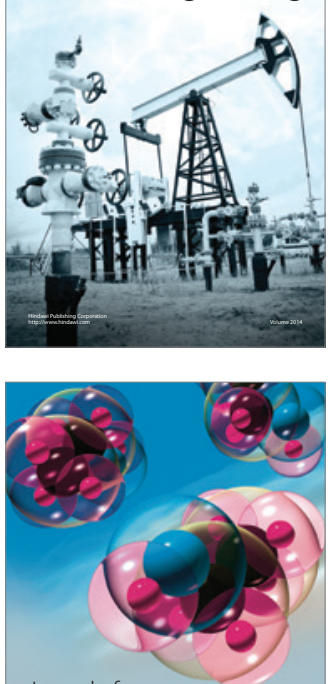

Fuels
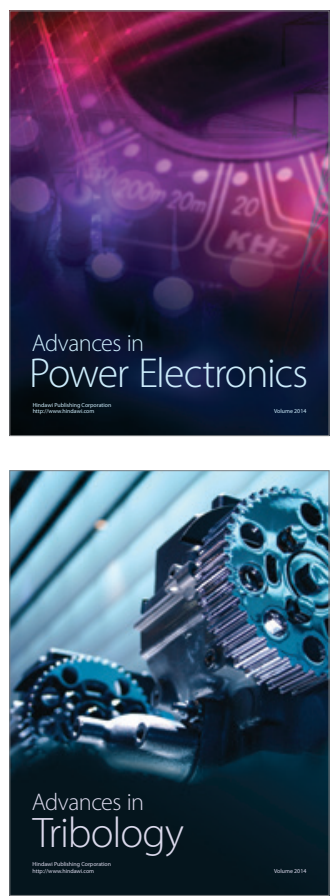

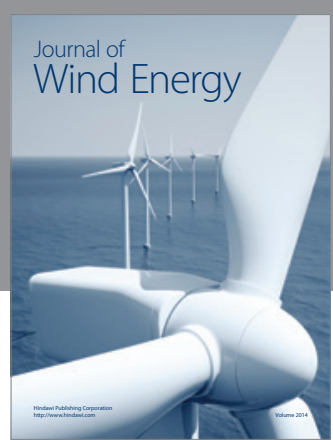

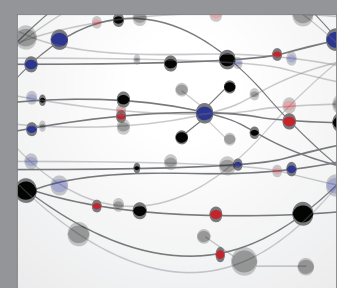

The Scientific World Journal

Submit your manuscripts at http://www.hindawi.com

Journal of

Structures
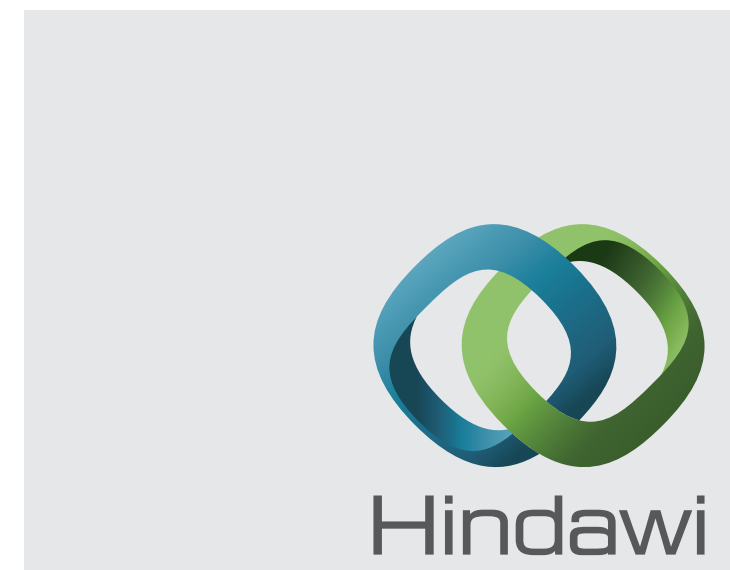

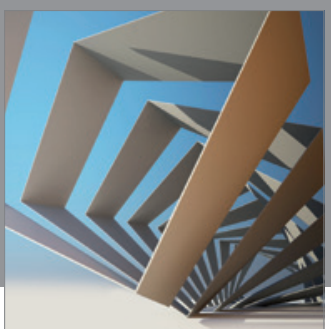

Rotating

Machinery
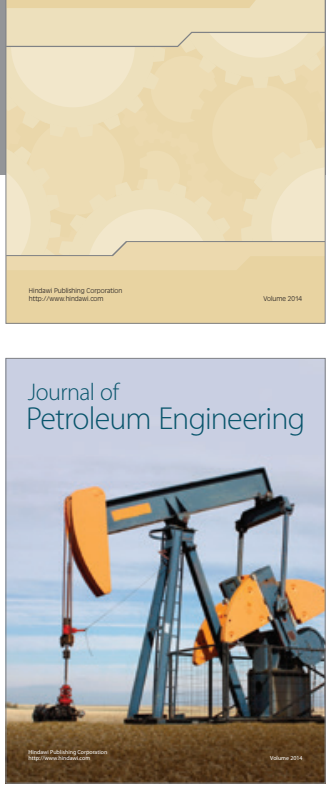

Journal of

Solar Energy
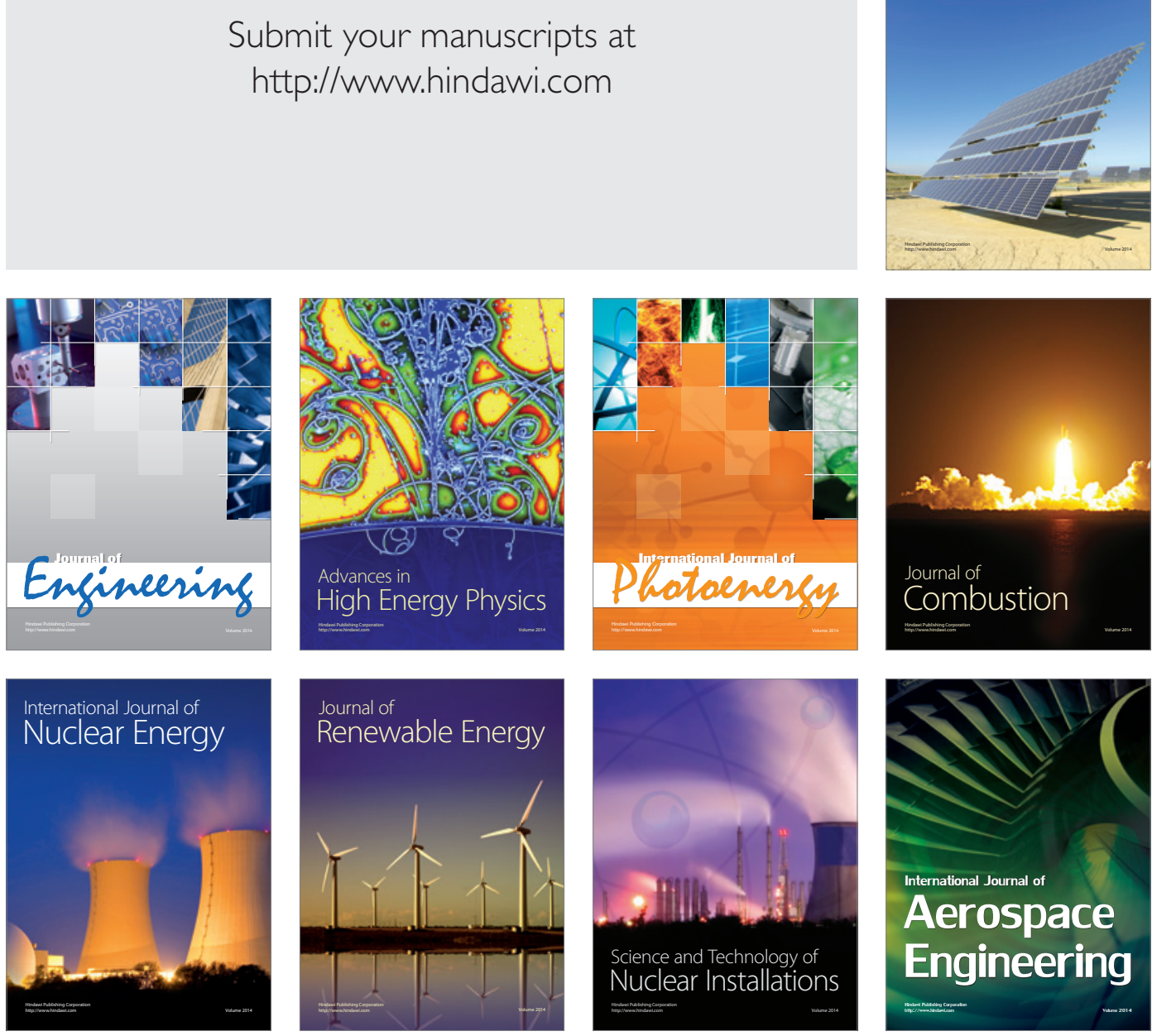